\title{
Graphene as a nanoelectromechanical reference piezoresistor
}

\author{
Abhinaba Sinha $\odot,{ }^{1}$ Abhishek Sharma, ${ }^{2}$ Pankaj Priyadarshi $\odot,{ }^{1}$ Ashwin Tulapurkar, ${ }^{1}$ and Bhaskaran Muralidharan $\oplus^{1}$ \\ ${ }^{1}$ Department of Electrical Engineering, IIT Bombay, Powai, Mumbai-400076, India \\ ${ }^{2}$ Department of Electrical Engineering, IIT Ropar, Rupnagar-140001, India
}

(Received 30 June 2020; accepted 14 September 2020; published 8 October 2020)

\begin{abstract}
Motivated by the recent prediction of anisotropy in piezoresistance of ballistic graphene along longitudinal and transverse directions, we investigate the angular gauge factor of graphene in the ballistic and diffusive regimes using highly efficient quantum transport models. It is shown that the angular gauge factor in both ballistic and diffusive graphene between $0^{\circ}$ to $90^{\circ}$ bears a sinusoidal relation with a periodicity of $\pi$ due to the reduction of sixfold symmetry into a twofold symmetry as a result of applied strain. The angular gauge factor is zero at critical angles $20^{\circ}$ and $56^{\circ}$ in ballistic and diffusive regimes, respectively. Based on these findings, we propose a graphene-based ballistic nanosensor which can be used as a reference piezoresistor in a Wheatstone bridge readout technique. The reference sensors proposed here are unsusceptible to inherent residual strain present in strain sensors and unwanted strain generated by the vapors in explosives detection. The theoretical models developed in this paper can be applied to explore similar applications in other two-dimensional Dirac materials. The proposals made here potentially pave the way for implementation of nanoelectromechanical strain sensors based on the principle of ballistic transport, which will eventually replace conventional microelectromechanical piezoresistance sensors with a decrease in feature size. The presence of strain-insensitive "critical angle" in graphene may be useful in flexible wearable electronics also.
\end{abstract}

DOI: 10.1103/PhysRevResearch.2.043041

\section{INTRODUCTION}

The development of microelectromechanical/nanoelectromechanical systems (MEMS/NEMS) has brought significant changes in every aspect of human life. The applications of MEMS in areas such as biotechnology [1,2], medicine $[3,4]$, avionics [5,6], particle transportation [7], and defense $[8,9]$ are virtually limitless. High-performance microscale systems, devices, and structures, including transducers $[10,11]$, switches [12,13], logic gates [14,15], actuators $[16,17]$, and sensors $[18,19]$, are currently used in day to day life. Graphene, a single-atom-thick material, possesses extraordinary electromechanical properties such as high elasticity $(\approx 20 \%)$ [20,21], Young's modulus $(\approx 1 \mathrm{TPa})$ [22], mobility [23], and mean-free path (in submicron range) [23-25]. Due to these properties, it is considered a promising material for next-generation microelectromechanical/nanoelectromechanical systems. Graphene is already used in MEMS systems as sensors [26-28], switches [29], resonators [30], and actuators [31,32], to name a few.

Rapid miniaturization of MEMS systems as a result of state-of-the-art nanofabrication techniques, on one hand, offers multiple applications in a single chip, and on the other, necessitates the revamping of the theoretical understanding

Published by the American Physical Society under the terms of the Creative Commons Attribution 4.0 International license. Further distribution of this work must maintain attribution to the author $(s)$ and the published article's title, journal citation, and DOI. of electronic transport processes at a microscopic level in both the ballistic [33-38] and the diffusive regimes [39,40]. A deeper theoretical understanding of electronic transport across these systems will hence lead to novel functionalities that govern the next-generation NEMS devices. In this work, we explore the use of graphene in strain sensing in both the ballistic and diffusive limits.

The piezoresistance of graphene in both ballistic and diffusive regimes has been studied previously by various groups [26,41-43]. The value of the gauge factor (GF) of ballistic and diffusive graphene for a uniaxial strain is reported in the range $0.3-6.1[26,41,43]$. In ballistic graphene, an anisotropy of the order of 10 exists between the longitudinal gauge factor (LGF) and the transverse gauge factor (TGF) [41]. Motivated by the presence of such an anisotropy in GF, we further venture to explore the variation of GF along different directions $\left(0^{\circ}\right.$ to $\left.90^{\circ}\right)$.

We devise a theoretical model using quantum transport theory built from the tight-binding representation to calculate the angular gauge factor (AGF) in the ballistic regime. Our model is highly efficient and thus reduces the computation time to $3 \%$ of that required by the conventional band-counting method in Ref. [41]. The theoretical model used in this paper can be extended to other two-dimensional (2D) Dirac materials $[44,45]$ as well. We obtain the AGF in the diffusive regime using the conductivity model developed by Peres et al. [46]. The value of GF simulated previously in the diffusive regime uses an approximation for Fermi velocity instead of the actual value $[41,42]$. In this work, we calculate the actual value of Fermi velocity along different directions which enables us to get an accurate value of AGF in the diffusive regime. We find 


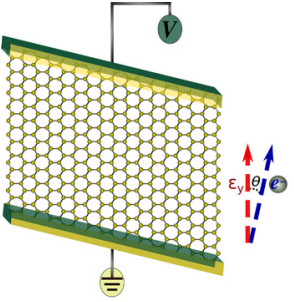

(a)

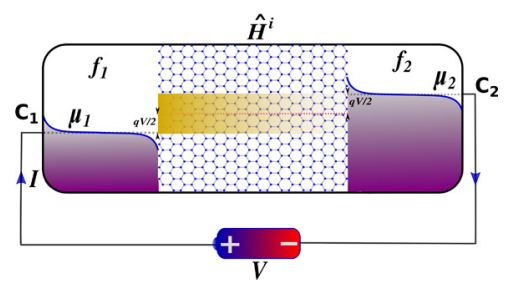

(b)
FIG. 1. Description of device schematic and transport setup for AFG calculation. (a) Schematic of a uniaxial strained graphene along the zigzag direction ( $y$ axis). The red and blue arrows represent the respective directions of applied strain and electron transport. The angle $\theta$ between them varies within the limit $(0, \pi / 2)$. (b) Voltagedriven charge transport model across a strained graphene sheet (with Hamiltonian $\hat{H}^{i}$, where $i$ denotes strain percentage) sandwiched between electronic contacts $C_{1}$ and $C_{2}$ with Fermi levels $\mu_{1}=\mu_{0}-$ $q V / 2$ and $\mu_{2}=\mu_{0}+q V / 2$, respectively. Transport formalism is restricted within the linear response regime.

that the AGF in ballistic and diffusive graphene is a sinusoidal function of the transport direction with a periodicity of $\pi$ due to the reduction of sixfold symmetry into twofold symmetry on the application of a uniaxial strain. The AGF becomes zero at the critical angles $20^{\circ}$ and $56^{\circ}$ in ballistic and diffusive graphene, respectively. Using these results, we propose a ballistic nanosensor and a reference resistor using graphene in a Wheatstone bridge based readout technique. Further, the proposals made here potentially pave the way for implementation of NEMS strain sensors based on the principle of ballistic transport, which will eventually replace conventional MEMS piezoresistance sensors with a decrease in feature size. The presence of strain-insensitive "critical angle" in graphene may be useful in flexible wearable electronics also.

In the subsequent sections, we develop the mathematical model to calculate the AGF of graphene across different transport regimes, explain the underlying physics for the predicted results, and discuss the applications and future scopes. The detailed derivation of mathematical expressions is given in the Appendix.

\section{THEORETICAL MODEL}

\section{A. Simulation setup}

The schematic diagram of the angular gauge factor setup is presented in Fig. 1(a). A uniaxial strain $\left(\varepsilon_{y}\right)$ is applied along the zigzag direction, and the resistance is computed along different transport directions represented by $\theta$.

The quantum transport model for the given setup is schematized in Fig. 1(b). The setup constitutes a graphene sheet described by Hamiltonian $\hat{H}^{i}$, and ideal reflectionless contacts $C_{1}$ and $C_{2}$. The equilibrium Fermi energy of the graphene sheet and contacts are maintained at $0 \mathrm{eV}$. The voltages applied at the terminals $C_{1}$ and $C_{2}$ are $-V / 2$ and $V / 2$ volts, respectively.

The simulation setup described here evaluates AGF in the linear regime $[-0.01-0.01 \mathrm{eV}]$ for a linear elastic strain [0\%-10\%].

\section{B. Angular gauge factor calculation}

The transport properties of graphene in the ballistic regime depend on mode density [33], whereas in the diffusive regime depends on Fermi velocity [46]. The value of mode density and Fermi velocity depend on the applied strain. We evaluate the mode density and Fermi velocity of graphene as a function of strain along different directions $(\theta)$ from the band structure of strained graphene. We derive the mathematical models using quantum transport and semiclassical transport formalisms to evaluate AGF in two different transport regimes of graphene.

\section{Tight-binding model}

The tight-binding Hamiltonian of a honeycomb lattice is expressed as

$$
\hat{H}^{i}=\sum_{l, \tau} t_{\tau}^{i} c_{l} c_{\tau}^{\dagger}+\text { H.c. }
$$

In Eq. (1), $t_{\tau}^{i}$ represents the hopping parameter that connects the lattice site $l$ with its neighbors $\tau$ in graphene at strain $\varepsilon_{y}=i \% . c_{l}$ and $c_{\tau}^{\dagger}$ are, respectively, the annihilation and creation operators of electrons at sites $l$ and $\tau$. We consider that the electron dynamics of graphene is governed by the nearest-neighbor tight-binding Hamiltonian. Thus, the energy eigenvalues of Eq. (1) are given by

$$
E^{i}(k)= \pm\left|t_{1}^{i} e^{-j \vec{k} \cdot \vec{a}_{1}^{i}}+t_{2}^{i}+t_{3}^{i} e^{-j \vec{k} \cdot \vec{a}_{2}^{i}}\right|,
$$

where $\overrightarrow{a_{1}^{i}}$ and $\overrightarrow{a_{2}^{i}}$ are the basis vectors of strained graphene, and $t_{1}^{i}, t_{2}^{i}$, and $t_{3}^{i}$ are the nearest-neighbor hopping parameters.

We obtain the tight-binding parameters of uniaxially strained graphene from Ribeiro et al. [47]. Ribeiro obtains the parameters by fitting Eq. (2) with $a b$ initio band structures. This model is valid for energy $E$ in the range $[-0.2-0.2 \mathrm{eV}]$.

The nearest-neighbor tight-binding model of graphene described by Eq. (2) accurately predicts the shift in Dirac cones due to strain, band-gap threshold, and anisotropy in Fermi velocity $[48,49]$. This model is consistent with ab initio calculations [42,47,50-52] and experiments [21,53]. Thus, Eq. (2) is suitable for AGF calculation in the linear regime.

\section{Ballistic regime}

We compute the current-voltage characteristics of graphene in ballistic regime using Landauer formula which is expressed as

$$
I_{\theta}^{i}(V)=\frac{2 q}{h} \int_{-\infty}^{\infty} M_{\theta}^{i}(E)\left[f_{1}\left(E-\mu_{1}\right)-f_{2}\left(E-\mu_{2}\right)\right] d E,
$$

where $M_{\theta}^{i}(E)$ is the mode density at energy $E$, percentage strain $i$, and electron transport direction $\theta$. The resistance calculated from Eq. (3) is expressed as

$$
R_{\theta}^{i}=\frac{1}{d\left\{I_{\theta}^{i}(V)\right\} / d V} .
$$




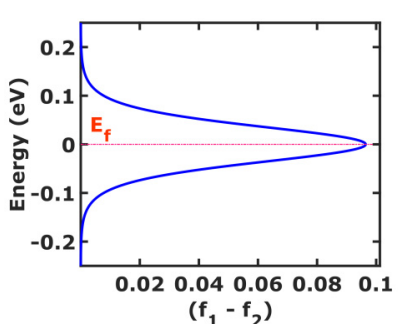

(a)

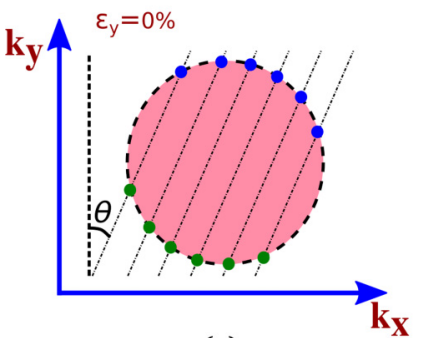

(c)

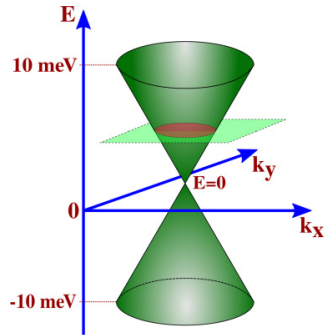

(b)

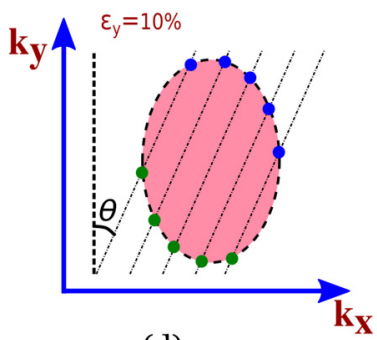

(d)
FIG. 2. Validation of the tight-binding model and mode density calculation in ballistic graphene. (a) Depiction of the Fermi window $\left(f_{1}-f_{2}\right)$ as a function of energy predefined in Fig. 1(b). The window opens up in the energy range $[-0.2 \mathrm{eV}, 0.2 \mathrm{eV}]$. (b) $3 \mathrm{D}$ view of graphene band structure close to the Dirac point. (c), (d) Depict the constant energy surface and modes along $\theta$. Constant energy surface shapes like (c) a circle for $0 \%$ strain and like (d) an oval for $10 \%$ strain, respectively. In each case, modes are depicted by blue dots.

The expression for AGF in ballistic regime averaged over the entire linear elastic limit is written as

$$
\begin{aligned}
(\mathrm{AGF})_{\theta}^{\mathrm{i}} & =\left\{\frac{R_{\theta}^{i}-R_{\theta}^{0}}{\varepsilon_{y} R_{\theta}^{0}}\right\}, \\
(\mathrm{AGF})_{\theta} & =\overline{(\mathrm{AGF})_{\theta}^{i}} .
\end{aligned}
$$

The Fermi window $f_{1}\left(E-\mu_{1}\right)-f_{2}\left(E-\mu_{2}\right)$ depends on the energy and applied voltage. We apply a variable potential difference between -0.01 to $0.01 \mathrm{~V}$ across the contacts. The Fermi-window plot as a function of energy at $0.01 \mathrm{~V}$ is shown in Fig. 2(a). The Fermi window is nonzero between -0.2 to $0.2 \mathrm{eV}$. Thus, our model using Eqs. (2) and (3) can accurately determine the angular piezoresistance of graphene in linear regime.

We obtain the mode density $M_{\theta}^{i}(E)$ from band structures under the Dirac cone approximation. Figure 2(b) illustrates the constant energy surface formed as a result of intersection of the constant energy plane and the Dirac cone. Figures 2(c) and 2(d) illustrate the constant energy surface and modes along an arbitrary transport direction $\theta$ at $0 \%$ and $10 \%$ strain. The blue and green dots represent the modes for forward and backward moving electrons, respectively. Since the effective number of Dirac cones within the first Brillouin zone of strained and unstrained graphene is two [41]. Thus, the effective number of modes at energy $E$ along transport direction $\theta$ is numerically equal to the sum of modes of forward and backward moving electrons in a single Dirac cone. As a result, the mode density is given by

$$
M_{\theta}^{i}(E)=2 n_{\theta}^{i}(E)
$$

where $n_{\theta}^{i}(E)$ is the number of transverse modes (TMs) intersecting the constant energy surface at energy $E$. The separation between TMs in Figs. 2(c) and 2(d) is $2 \pi / w_{\theta}^{i}$ where $w_{\theta}^{i}$ is the width of graphene sheet (see Appendix A).

The theoretical model described above (modified bandcounting method) reduces the computation time significantly compared to full band mode density calculation (bandcounting method) [41]. A comparison between the bandcounting method [41,54], and the modified band-counting method used in this paper is given in Table I.

\section{Diffusive regime}

The electron transport in diffusive regime is described via the semiclassical transport mechanism. The electrons are treated as classical particles (wave packets) whose position and momentum are precisely known [55]. The electron mobility primarily determines the electron transport properties in the diffusive regime as compared to the mode density in ballistic regime.

The experimentally determined conductivity of graphene in the diffusive regime shows a linear dependence on electron density except at the charge neutrality point [23]. By considering random Coulomb impurities as the dominant source of scattering, a linearly varying conductivity with gate voltage is obtained in Ref. [56]. The expression for conductivity of graphene as a function of the electron density, considering the presence of charged impurities was formulated using the Boltzman transport theory under relaxation time approximation by Peres et al. [46]. The expression for conductivity as derived in Ref. [46] is given by

$$
\sigma=\frac{2 e^{2} \pi\left(\hbar v_{f}\right)^{2} n}{h u_{o}^{2}} .
$$

The conductivity of graphene in diffusive regime depends on the electron density and the Fermi velocity. However, variation in the conductivity due to a change in electron density is prominent only in the presence of a gate voltage [49]. In the absence of gate voltage, conductivity depends primarily on Fermi velocity. The anisotropy in resistance due to a tensile strain predicted using Eq. (7) complies with the experimental results [21]. Hence, we use Eq. (7) to obtain the expression for AGF in diffusive regime.

The resistance of a uniaxially strained graphene is given by

$$
R_{\theta}^{i}=\frac{l_{\theta}^{i}}{\sigma_{\theta}^{i} w_{\theta}^{i}},
$$

where $R_{\theta}^{i}, \sigma_{\theta}^{i}, l_{\theta}^{i}$, and $w_{\theta}^{i}$ are the resistance, conductivity, length, and width, respectively, at percentage strain $i$ along the direction $\theta$.

Thus, the expression for AGF in diffusive regime averaged over the entire strain range is given by

$$
\begin{aligned}
(\mathrm{AGF})_{\theta}^{\mathrm{i}} & =\frac{1}{\varepsilon_{y}}\left\{\frac{\Delta l_{\theta}^{i}}{l_{\theta}^{0}}-\frac{\Delta w_{\theta}^{i}}{w_{\theta}^{0}}-2 \frac{\Delta v_{\theta}^{i}}{v_{\theta}^{0}}\right\}, \\
(\mathrm{AGF})_{\theta} & =\frac{(\mathrm{AGF})_{\theta}^{i}}{}
\end{aligned}
$$

The AGF in diffusive regime depends on the variation of Fermi velocity, electron density, and dimensions of the graphene with strain $\varepsilon_{y}$ and direction $\theta$. The strain-induced 
TABLE I. Comparison between the band-counting and modified band-counting methods.

\begin{tabular}{lll}
\hline \hline Methods & \multicolumn{1}{c}{ Conventional band counting } & Modified band counting \\
\hline Application & 2D materials & 2D Dirac materials \\
Approximation/assumption & Identical TMs in each segments of Brillouin zone & Dirac-cone approximation \\
Range of validity & Full band structure & Linear regime \\
Quality of prediction & GF consistent with earlier works & Same as band-counting method \\
Computation time & Less efficient & Highly efficient \\
\hline
\end{tabular}

variation of Fermi velocity is discussed in this section, while the strain-induced change in dimensions is discussed separately in the next subsection.

The velocity of electrons at an energy close to the Dirac point is equal to its Fermi velocity. The Fermi velocity is expressed as

$$
v_{\phi}^{i}=\left.\frac{1}{\hbar}\left\{\nabla_{k} E^{i}(k)\right\}\right|_{k=k_{\phi}^{i}} .
$$

Figures 3(a) and 3(b) show the Fermi-velocity vectors and contours near a Dirac point at $0 \%$ and $10 \%$ strain, respectively. The variation in magnitude of Fermi velocity with $\varepsilon_{y}$ and $\theta$ is given in Table II. From the table, we infer that strain induces anisotropy in the Fermi velocity. The AGF in diffusive

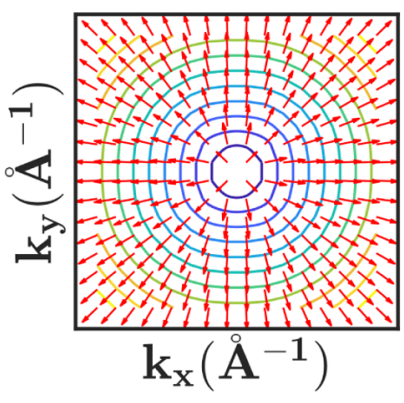

(a)

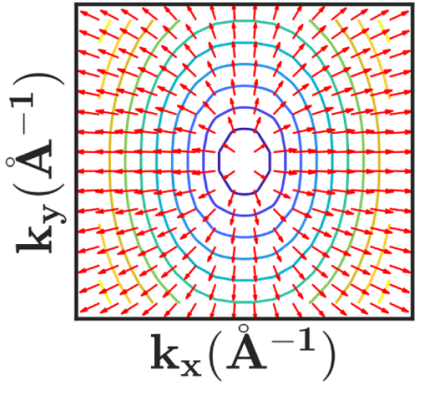

(b)

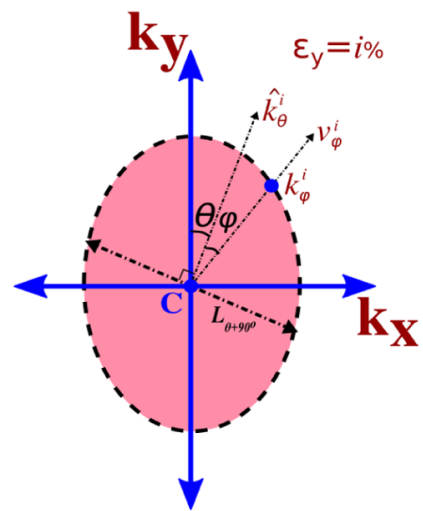

(c)

FIG. 3. Calculation of average Fermi velocity in graphene for computing AGF in the diffusive regime. (a), (b) Depict the Fermivelocity vectors (represented by the red arrows) and energy contours near the Dirac point for $\varepsilon_{y}=0 \%$ and $10 \%$, respectively. (b) Shows that strain induces anisotropy in Fermi velocity. (c) Depicts the schematics of the unit vector $k_{\theta}^{i}$ along $\theta$ and Fermi velocity $v_{\phi}^{i}$ along $\phi$. regime depends on the average Fermi velocity along the direction of transport. Figure 3(c) illustrates the methodology for evaluation of average Fermi velocity along the transport direction $(\theta)$. The average Fermi velocity along $\theta$ at strain $i$ is expressed as

$$
\overline{v_{\theta}^{i}}=\frac{1}{\pi} \int_{-\frac{\pi}{2}}^{\frac{\pi}{2}}\left\{v_{\phi}^{i} \cdot \hat{k_{\theta}^{i}}\right\} d \phi,
$$

where $v_{\phi}^{i}$ is the Fermi velocity along direction $\phi$ and $\hat{k_{\theta}^{i}}$ is the unit vector along $\theta$. See Appendix B for detailed derivation of Eq. (11).

\section{Strain distribution in graphene}

Apart from the variation of Fermi velocity, AGF also depends on the magnitude of strain along the transport $(\theta)$ and transverse directions $\left(90^{\circ}+\theta\right)$. The changes in dimensions modify the mode density and conductivity of graphene [see Eqs. (6) and (9)].

The strain $\varepsilon_{y}$ generates components along different directions of graphene. The stiffness or compliance matrix due to a uniaxial strain along the basal plane of the graphene sheet is the same irrespective of the choice of coordinate axes [57]. Consequently, the Poisson's ratio of graphene sheet is same irrespective of the direction of tensile strain in the basal plane [49].

The mean-free path of graphene is very high and is in the submicron range [23-25]. Therefore, we treat graphene as a continuum sheet in strain-related calculations in this work. The strain components along the electron transport direction $\left(\varepsilon_{\theta}\right)$ and its transverse direction $\left(\varepsilon_{\theta}^{\dagger}\right)$ are expressed as [57]

$$
\begin{aligned}
\varepsilon_{\theta} & =\frac{1}{2}\left(\varepsilon_{x}+\varepsilon_{y}\right)+\frac{1}{2}\left(\varepsilon_{y}-\varepsilon_{x}\right) \cos 2 \theta, \\
\varepsilon_{\theta}^{\dagger} & =\frac{1}{2}\left(\varepsilon_{x}+\varepsilon_{y}\right)+\frac{1}{2}\left(\varepsilon_{y}-\varepsilon_{x}\right) \cos 2\left(\theta+90^{\circ}\right),
\end{aligned}
$$

where $\varepsilon_{y}$ is the longitudinal strain and $\varepsilon_{x}$ is the transverse strain $\left(-\sigma \varepsilon_{y}\right)$. In the ballistic regime, mode density depends on the separation between two adjacent TMs which is given by $2 \pi / w_{\theta}^{i}$, where $w_{\theta}^{i}=w_{\theta}^{0}\left(1+\varepsilon_{\theta}^{\dagger}\right)$. In the diffusive regime,

TABLE II. Variation of Fermi velocity (in the scale of $\times 10^{5} \mathrm{~m} / \mathrm{s}$ ) with different values of strain $\left(\varepsilon_{y}\right)$ and transport angle $(\theta)$.

\begin{tabular}{lcccc}
\hline \hline$\varepsilon_{y}$ & $0^{\circ}$ & $30^{\circ}$ & $60^{\circ}$ & $90^{\circ}$ \\
\hline $0 \%$ & 8.41 & 8.41 & 8.41 & 8.41 \\
$5 \%$ & 7.35 & 7.75 & 8.37 & 8.59 \\
$10 \%$ & 6.12 & 7.32 & 8.45 & 8.77 \\
\hline \hline
\end{tabular}




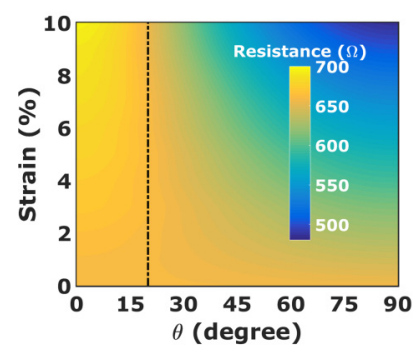

(a)

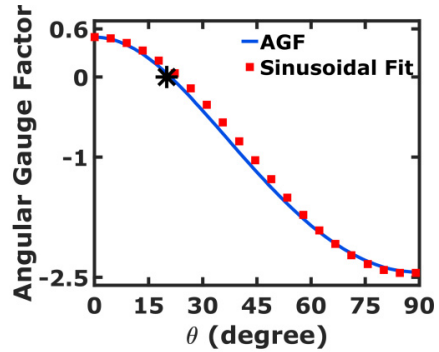

(b)
FIG. 4. Piezoresistance along different transport directions in the ballistic graphene. (a) Color map of resistance of a 1- $\mu \mathrm{m}$-wide graphene sheet as a function of $\varepsilon_{y}$ and $\theta$. (a) Shows that the resistance remains constant at $\theta=20^{\circ}$. (b) Depicts AGF along with its sinusoidal fit. It infers zero AGF at $\theta=20^{\circ}$.

apart from Fermi velocity, AGF varies with $\varepsilon_{\theta}$ and $\varepsilon_{\theta}^{\dagger}$ also [see Eq. (9)].

\section{RESULTS AND DISCUSSIONS}

In this section, we obtain the AGF of graphene using the theoretical models discussed earlier and obtain a suitable mathematical fit. We discuss the physics behind the predicted results along with their applications and prospects.

We show in Fig. 4(a) the variation of resistance of a $1-\mu \mathrm{m}-$ wide ballistic graphene sheet with $\varepsilon_{y}$ and $\theta$. The resistance at $0 \%$ strain is constant irrespective of the direction $\theta$. Nevertheless, the variation in resistance increases with $\theta$ as $\varepsilon_{y}$ increases. Thus, Fig. 4(a) validates the fact that graphene is electrically isotropic at $0 \%$ strain and becomes anisotropic on the application of tensile strain $[41,49]$. The anisotropy increases with an increase in strain. The resistance along $\theta=0^{\circ}$ increases by a small amount with an increase in strain. However, it decreases significantly along $\theta=90^{\circ}$ with an increase in strain. We note that the resistance remains constant with applied strain at $\theta=20^{\circ}$. We show in Fig. 4(b) the variation of AGF with $\theta$ and the corresponding sinusoidal fit which is a sinusoidal function of the form

$$
\mathrm{AGF}=A \cos 2 \theta+B,
$$

where $A=1.475$ and $B=-0.975$. We see in Fig. 4(b) that the AGF along $0^{\circ}, 20^{\circ}$, and $90^{\circ}$ are $0.6,0$, and -2.5 , respectively. The observed pattern of AGF in the ballistic regime is a result of the deformation of Dirac cone and change in separation of TMs due to strain. We report a GF of 0.5 at the terminal angle $\theta=0^{\circ}$ in the ballistic regime, as compared to a GF of 0.3 reported in Ref. [41]. The transmissions along $\theta=20^{\circ}$ at different strains are identical as shown in Fig. 5, thereby substantiating our claim of resistance invariance along that direction.

We show in Fig. 6(a) the variation of average Fermi velocity with $\theta$ and $\varepsilon_{y}$. The average Fermi velocity is constant at $0 \%$ strain along differnt directions $\theta$ and has a magnitude of $5.35 \times 10^{5} \mathrm{~m} / \mathrm{s}$. The average Fermi velocity decreases sharply along $\theta=0^{\circ}$, becomes zero $\theta \approx 60^{\circ}$, and finally slightly increases along $90^{\circ}$ with the increase in strain. The variation in average Fermi velocity with $\varepsilon_{y}$ and $\theta$ is similar to the variation of Fermi velocity (see Table II). Figures 6(b) and 6(c) depict

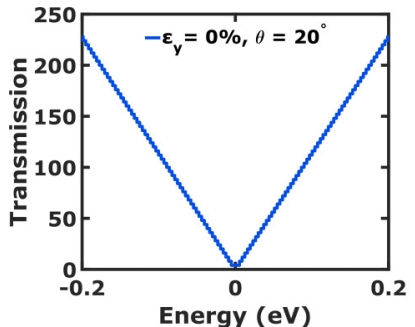

(a)

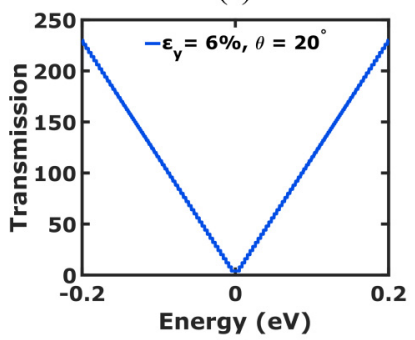

(c)

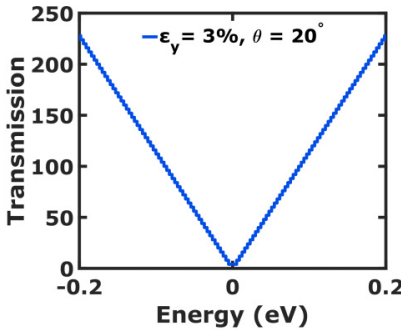

(b)

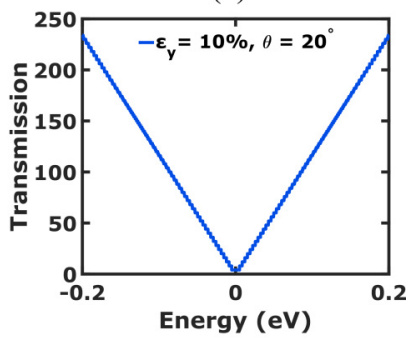

(d)
FIG. 5. Variation of transmission as a function of energy within the Fermi window at different strains $\varepsilon_{y}$ keeping the transport angle $\theta$ fixed at $20^{\circ}$.

$\varepsilon_{\theta}$ and $\varepsilon_{\theta}^{\dagger}$ as a function of $\varepsilon_{y}$ and $\theta$. The color maps of $\varepsilon_{\theta}$ and $\varepsilon_{\theta}^{\dagger}$ are mirror images of each other due to twofold symmetry and isotropic nature of a uniaxially strained graphene (explained in the next paragraph).

Figure 6(d) presents the AGF in diffusive graphene. The plot of AGF with $\theta$ can be approximated by a sinusoidal curve given by

$$
\mathrm{AGF}=C \cos 2 \theta+D,
$$

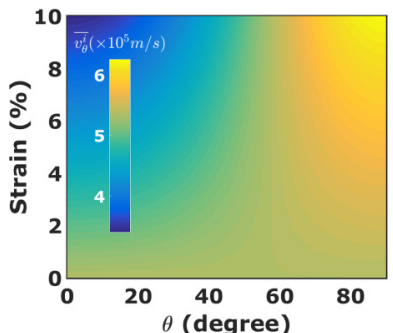

(a)

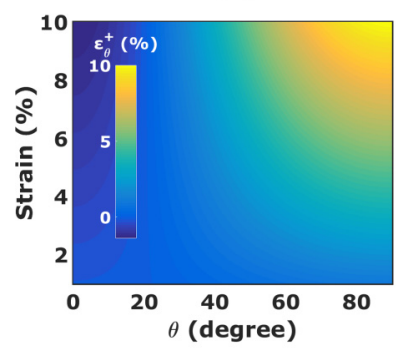

(c)

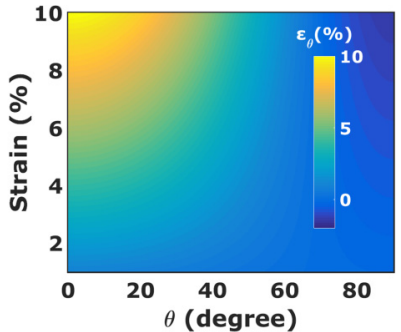

(b)

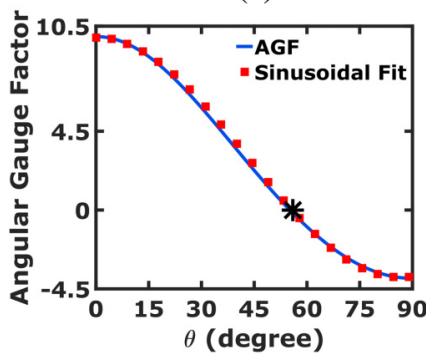

(d)
FIG. 6. Study of the parameters determining the AGF in diffusive regime. (a) Color map of the average Fermi velocity as a joint function of strain $\left(\varepsilon_{y}\right)$ and transport angle $(\theta)$. Color map of strain along (b) the transport direction and (c) the transverse direction as a function of $\epsilon_{y}$ and $\theta$, respectively. (d) Depicts the AGF and its sinusoidal fit as a function of $\theta$. 


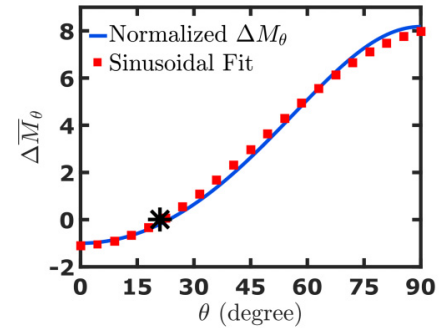

(a)

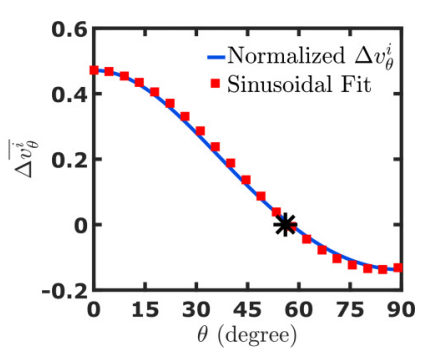

(b)
FIG. 7. Variation of the (a) normalized mode density (in case of ballistic graphene) and (b) average Fermi velocity (in case of diffusive graphene) as a function of transport direction $(\theta)$ and their sinusoidal fit. (a), (b) Provide explanation for the sinusoidal variation of AGF with $\theta$.

where $C=6.85$ and $D=3$. The value of AGF varies sinusoidally between 9.85 to -3.85 and is zero at $56^{\circ}$ in the diffusive regime. The GF in diffusive graphene reported earlier is in the range 2.4-6.73 [26,42] as compared to a GF of 9.85 obtained in this paper. We see that ballistic graphene has higher GF along $\theta=90^{\circ}$ whereas diffusive graphene has higher GF along $\theta=0^{\circ}$.

Graphene has a sixfold symmetry, but due to the application of tensile strain, its symmetry reduces to a twofold symmetry. In Figs. 4(b) and 6(d), the AGF plots have a periodicity of $\pi$ which is due to the twofold symmetry of uniaxially strained graphene lattice.

The results obtained in this paper are for a strain applied along the zigzag direction. Nevertheless, the results are same for strain along the armchair direction due to the isotropic Poisson's ratio [49] in the basal plane and identical deformation of the Dirac cone for strain along with armchair and zigzag directions [41]. The explanation for sinusoidal AGF and the application of these results are discussed in the subsequent sections of this paper.

\section{A. Physics of sinusoidal AGF}

The mode density along transport angle $\theta$ changes as a result of the applied strain $\varepsilon_{y}$ due to the deformation of Dirac cone [41]. The change in mode density is inversely proportional to the change in normalized resistance along the transport angle $\theta$. In other words, AGF is inversely proportional to the change in normalized mode density. The mode density and change in the normalized mode density averaged over the entire strain range [0\%-10\%] is mathematically expressed as

$$
\begin{aligned}
M_{\theta}(E) & =\frac{w_{\theta} * L_{\theta+90^{\circ}}(E)}{2 \pi}, \\
\frac{\Delta M_{\theta}(E)}{M_{\theta}(E)} & =\frac{\Delta w_{\theta}}{w_{\theta}}+\frac{\Delta L_{\theta+90^{\circ}}(E)}{L_{\theta+90^{\circ}}(E)},
\end{aligned}
$$

where $M_{\theta}(E), w_{\theta}$, and $L_{\theta+90^{\circ}}(E)$ are, respectively, the mode density, width of the graphene sheet, and length of the axis of Dirac cone along the transverse direction $90^{\circ}+\theta$ as shown in Fig. 3(c). We show in Fig. 7(a) the plot of $\Delta \bar{M}_{\theta}$ represented by Eq. (15b). Figure 7(a) is similar to the reciprocal of AGF in Fig. 4(b). Thus, the sinusoidal nature of AGF in ballistic
TABLE III. Variation of normalized average Fermi velocity, strain along the transport direction $\theta$, and width along the transverse direction $\left(\theta+90^{\circ}\right)$.

\begin{tabular}{lrrr}
\hline \hline$\theta$ & $-2 \Delta \bar{v}_{\theta}$ & $\Delta \bar{L}_{\theta}$ & $-\Delta \bar{w}_{\theta}$ \\
\hline $0^{\circ}$ & 0.52 & 0.055 & 0.008 \\
$45^{\circ}$ & 0.13 & 0.024 & -0.024 \\
$90^{\circ}$ & -0.15 & -0.008 & -0.055 \\
\hline \hline
\end{tabular}

graphene is due to the sinusoidal variation of mode density along different $\theta$.

The AGF in diffusive graphene depends on the average Fermi velocity along $\theta$, strain along the transport direction $\left(\varepsilon_{\theta}\right)$, and the transverse direction $\left(\varepsilon_{\theta}^{\dagger}\right)$. Table III shows the variation of these parameters at transport angles $0^{\circ}, 45^{\circ}$, and $90^{\circ}$. From the table, we infer that AGF predominantly depends on the average Fermi velocity. We show in Fig. 7(b) the variation of average Fermi velocity with $\theta$ is similar to a sinusoidal function and resembles the AGF in diffusive regime. Thus, the sinusoidal variation of AGF with transport angle $\theta$ is due to the sinusoidal variation of average Fermi velocity with transport angle $\theta$.

\section{B. Application and future scope}

Piezoresistance sensor uses a Wheatstone bridge readout technique for the detection of strain. We show in Fig. 8(a) a Wheatstone bridge based piezoresistance sensing setup using ballistic graphene. The Wheatstone bridge consists of identical ballistic graphene resistors $R_{1}, R_{2}, R_{3}$, and $R_{4}$. At zero strain, the resistances are equal, and the Wheatstone bridge is balanced. In this configuration, $R_{4}$ acts as a strain sensor, while the other resistors act as reference resistors. When subjected to a strain, the resistance $R_{4}$ changes, which results in the generation of a potential difference $\left(V_{\text {out }}\right)$ [see Fig. 8(b)]. Figure 8(b) shows $V_{\text {out }}$ versus strain for different values of $\theta$ when the input voltage $V_{\text {in }}$ is maintained at $0.02 \mathrm{eV}$. Besides, $V_{\text {out }}$ exhibits a linear dependence on strain along different transport directions $\theta$ and is maximum at $\theta=90^{\circ}$.

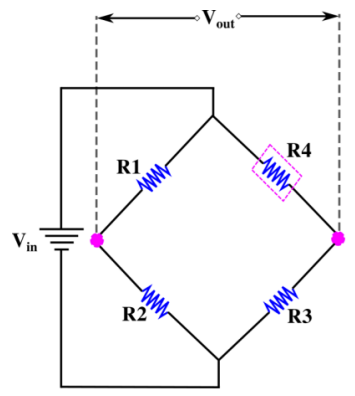

(a) (b)

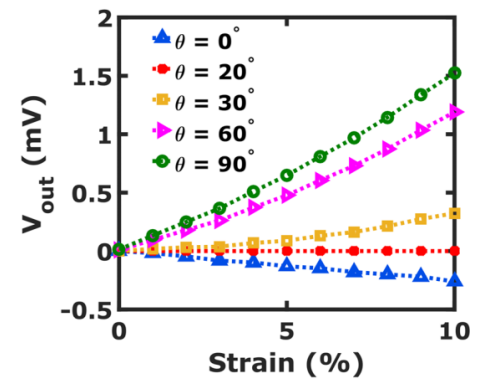

FIG. 8. Setup for piezoresistance sensing of a graphene-based ballistic nanosensor using the (a) Wheatstone bridge readout technique. $R_{3}$ and $R_{4}$ are the reference and the strain sensors, respectively. (b) Variation in the output voltage $\left(V_{\text {out }}\right)$ of the Wheatstone bridge with strain $\left(\varepsilon_{y}\right)$ and transport direction $(\theta)$. $V_{\text {out }}$ varies linearly with the strain along different directions. 
This indicates a high-strain sensitivity of ballistic graphene in the transverse configuration which can be used to detect explosives and gases provided the strain generated is mapped with the vapor density of explosives and gases [58-61].

A significant problem of strain sensor is the presence of an inherent residual strain. As a result, $V_{\text {out }}$ is nonzero, even in the absence of an external strain. Besides, the reference sensors in explosive or gas detectors are also vulnerable to unwanted strain produced by the explosive's vapor or gases [58-61]. We address these problem without removing the residual strain or unwanted strain generated during the detection.

As discussed earlier, the resistance of unstrained graphene is the same along every direction in the basal plane and remains the same even in the presence of strain when electron transport is directed along the critical angle. Based on this fact, we propose a graphene-based reference piezoresistor with electron transport direction along the critical angle such that the resistance remains steady and equal to that of the unstrained graphene.

The modified band-counting method used in this paper for graphene is also applicable to other 2D Dirac materials for similar applications. Some of the prominent 2D Dirac materials apart from graphene are silicene, germanene, stanene, several graphynes, just to name a few [62]. The modified band-counting method can be used to obtain the transport and piezoresistance properties by using a more generalized form of Eq. (6) given by

$$
M(E)=a \times n(E),
$$

where $a$ is the effective number of Dirac cones within the first Brillouin zone. Using Eq. (16) and the Landauer formula, piezoresistance properties of other 2D Dirac materials can be computed. In the case of buckled honeycomb 2D Dirac materials such as silicene, germanene, etc., the value of $a$ is two due to the presence of two effective Dirac cones in the first Brillouin zone [41]. In the case of graphynes having Dirac cones, the value of $a$ generally varies from two to six [62,63].

The excellent electromechanical properties of graphene make it a strong contender for materials used in flexible electronics $[21,64]$. The critical angles obtained in this paper may find application in future flexible devices where a constant current is required despite the presence of a variable strain.

\section{CONCLUSION}

In summary, we investigated the angular gauge factor of graphene in the ballistic and diffusive regimes using highly efficient quantum transport models. It was shown that the angular gauge factor in both ballistic and diffusive graphene between $0^{\circ}$ to $90^{\circ}$ bears a sinusoidal relation with a periodicity of $\pi$ due to the reduction of sixfold symmetry into twofold symmetry as a result of applied strain. The angular gauge factor is zero at critical angles $20^{\circ}$ and $56^{\circ}$ in ballistic and diffusive regimes, respectively. Based on these findings, we propose a graphene-based ballistic nanosensor, which can be used as a reference piezoresistor in a Wheatstone bridge readout technique. The reference sensors proposed here are unsusceptible to inherent residual strain present in strain sensors and unwanted strain generated by the vapors in explosives detection. The theoretical models developed in this paper can be applied to explore similar applications in other 2D Dirac materials. The proposals made here potentially pave the way for implementation of NEMS strain sensors based on the principle of ballistic transport, which will eventually replace MEMS piezoresistance sensors with a decrease in feature size. The presence of strain-insensitive "critical angle" in graphene may be useful in flexible wearable electronics also.

\section{ACKNOWLEDGMENTS}

The Research and Development work undertaken in the project under the Visvesvaraya Ph.D. Scheme of Ministry of Electronics and Information Technology, Government of India, is implemented by Digital India Corporation (formerly Media Lab Asia). This work was also supported by the Science and Engineering Research Board (SERB), Government of India, Grant No. EMR/2017/002853 and Grant No. STR/2019/000030, the Ministry of Human Resource and Development (MHRD), Government of India, Grant No. STARS/APR2019/NS/226/FS under the STARS scheme, and the Department of Science and Technology, Government of India under the Quantum Enabled Science and Technology (Quest) scheme, Project Code: RD/0119-DST0001-004. The author Abhinaba Sinha was also supported by NNetRA project under Sanction Order: DST/NM/NNetRA/2018(G)IIT-B at IIT Bombay.

\section{APPENDIX A: SEPARATION BETWEEN ADJACENT TRANSVERSE MODES IN A SHEET}

The separation between adjacent $k$ states of conduction electrons in reciprocal space is determined by periodic boundary condition [55]. Electrons in conduction band behave as nearly free electrons. The wave function of these electrons is expressed as

$$
\psi=A e^{i k \cdot r} .
$$

Equation (A1) represents a plane-wave equation traveling along $r$. Let us consider a crystal of length $L$ and wave function at $r=0$ and $L$ be denoted by $\psi_{0}$ and $\psi_{L}$, respectively. The wave functions at the boundaries are equal as a result of periodic boundary condition. Thus,

$$
\psi_{0}=\psi_{L} .
$$

Using Eqs. (A1) and (A2), we obtain the allowed $k$ state which is expressed as

$$
k=\frac{2 n \pi}{L},
$$

where $n=0,1,2,3, \ldots$ The separation between $k$ states in reciprocal space is $2 \pi / L$, where $L$ is the length of the crystal. Extending the same reasoning along the width $(w)$, we obtain a separation of $2 \pi / w$ between adjacent transverse modes (TMs) along the width in reciprocal space.

\section{APPENDIX B: AVERAGE FERMI VELOCITY UNDER DIRAC CONE APPROXIMATION}

Under the Dirac cone approximation, the velocity of electrons along $\phi$ at different energies is equal to the 
Fermi velocity (see Fig. 3). The magnitude of Fermi velocity along different directions is equal in unstrained graphene. Application of a tensile strain $\left(\varepsilon_{y}\right)$ results in anisotropic Fermi velocity [see Fig. 3(b)]. As a result, evaluation of the conductivity of strained graphene using Eq. (7) will require the value of average Fermi velocity along $\theta$.

Figure 3(c) illustrates the methodology for calculating average Fermi velocity. The direction of Fermi velocity $(\phi)$ forming an angle between $-\frac{\pi}{2}$ to $\frac{\pi}{2}$ with respect to $\theta$ have velocity components along $\theta$. The Fermi velocity $\left(v_{\phi}^{i}\right)$ along $\phi$ is expressed as

$$
v_{\phi}^{i}=\left.\frac{1}{\hbar}\left\{\nabla_{k} E^{i}(k)\right\}\right|_{k=k_{\phi}^{i}} .
$$

The component of $v_{\phi}^{i}$ along $\theta$ is $v_{\phi}^{i} \cdot k_{\theta}^{i}$, where $\hat{k_{\theta}^{i}}$ is a unit vector along $\theta$. Thus, the mean of these components for different values of $\phi$ along $\theta$ is given by

$$
\overline{v_{\theta}^{i}}=\frac{1}{\pi} \int_{-\frac{\pi}{2}}^{\frac{\pi}{2}}\left\{v_{\phi}^{i} \cdot \hat{k}_{\theta}^{i}\right\} d \phi .
$$

[1] M. Madou, in Symposium on Design, Test, Integration and Packaging of MEMS/MOEMS 2003 (IEEE, Piscataway, NJ, 2003), p. 1.

[2] W. Wang and S. A. Soper, Bio-MEMS: Technologies and Applications (CRC Press, Boca Raton, FL, 2006).

[3] N.-C. Tsai and C.-Y. Sue, Sens. Actuators A 134, 555 (2007).

[4] C. Kleinstreuer, J. Li, and J. Koo, Int. J. Heat Mass Transf. 51, 5590 (2008)

[5] J. S. Jang and D. Liccardo, IEEE Aerospace Electron. Syst. Mag. 22, 30 (2007).

[6] J. Leclerc, IEEE Aerospace Electron. Syst. Mag. 22, 31 (2007).

[7] A. Desai, S.-W. Lee, and Y.-C. Tai, Sens. Actuators A 73, 37 (1999).

[8] W. C. Tang and A. P. Lee, MRS Bull. 26, 318 (2001).

[9] J. P. Siepmann, in Laser Radar Technology and Applications $X I$ (International Society for Optics and Photonics, Bellingham, WA, 2006), Vol. 6214, p. 621409.

[10] Y.-W. Nam and S.-h. Lee, Flexible MEMS transducer and manufacturing method thereof, and flexible MEMS wireless microphone, US Patent No. 6,967,362 (2005).

[11] A. C. Mcneil, G. Li, and D. N. Koury Jr, Single proof mass, 3 axis mems transducer, 2005, US Patent No. 6,845,670.

[12] G. M. Rebeiz and J. B. Muldavin, IEEE Microwave Mag. 2, 59 (2001).

[13] E. R. Brown, IEEE Trans. Microwave Theory Tech. 46, 1868 (1998).

[14] C.-Y. Tsai, W.-T. Kuo, C.-B. Lin, and T.-L. Chen, J. Micromech. Microeng. 18, 045001 (2008).

[15] C.-Y. Tsai and T.-L. Chen, J. Micromech. Microeng. 20, 095021 (2010).

[16] D. J. Bell, T. Lu, N. A. Fleck, and S. M. Spearing, J. Micromech. Microeng. 15, S153 (2005).

[17] N. J. Conway, Z. J. Traina, and S.-G. Kim, J. Micromech. Microeng. 17, 781 (2007).

[18] J. W. Reeds III, Y. W. Hsu, and P. C. Dao, Mems sensor with single central anchor and motion-limiting connection geometry, US Patent No. 6,513,380 (2003).

[19] M. Kitano, H. Sumi, and T. Moribe, Capacitance difference detecting circuit and mems sensor US Patent No. 7,119,550 (2006).

[20] F. Liu, P. Ming, and J. Li, Phys. Rev. B 76, 064120 (2007).

[21] K. S. Kim, Y. Zhao, H. Jang, S. Y. Lee, J. M. Kim, K. S. Kim, J.-H. Ahn, P. Kim, J.-Y. Choi, and B. H. Hong, Nature (London) 457, 706 (2009).

[22] C. Lee, X. Wei, J. W. Kysar, and J. Hone, Science 321, 385 (2008).
[23] K. S. Novoselov, A. K. Geim, S. V. Morozov, D. Jiang, Y. Zhang, S. V. Dubonos, I. V. Grigorieva, and A. A. Firsov, Science 306, 666 (2004).

[24] S. Adam and S. D. Sarma, Solid State Commun. 146, 356 (2008).

[25] A. H. Castro Neto, F. Guinea, N. M. R. Peres, K. S. Novoselov, and A. K. Geim, Rev. Mod. Phys. 81, 109 (2009).

[26] A. Smith, F. Niklaus, A. Paussa, S. Vaziri, A. C. Fischer, M. Sterner, F. Forsberg, A. Delin, D. Esseni, P. Palestri et al., Nano Lett. 13, 3237 (2013).

[27] R. J. Dolleman, D. Davidovikj, S. J. Cartamil-Bueno, H. S. van der Zant, and P. G. Steeneken, Nano Lett. 16, 568 (2015).

[28] Q. Wang, W. Hong, and L. Dong, Nanoscale 8, 7663 (2016).

[29] Y. Li, H. Yu, X. Qiu, T. Dai, J. Jiang, G. Wang, Q. Zhang, Y. Qin, J. Yang, and X. Jiang, Sci. Rep. 8, 1562 (2018).

[30] C. Chen, S. Rosenblatt, K. I. Bolotin, W. Kalb, P. Kim, I. Kymissis, H. L. Stormer, T. F. Heinz, and J. Hone, Nat. Nanotechnol. 4, 861 (2009).

[31] Y. Huang, J. Liang, and Y. Chen, J. Mater. Chem. 22, 3671 (2012).

[32] G. W. Rogers and J. Z. Liu, J. Am. Chem. Soc. 133, 10858 (2011).

[33] S. Datta, Lessons from Nanoelectronics: A New Perspective on Transport (World Scientific, Singapore, 2012).

[34] U. Patil and B. Muralidharan, Physica E (Amsterdam) 85, 27 (2017).

[35] P. Priyadarshi, A. Sharma, S. Mukherjee, and B. Muralidharan, J. Phys. D: Appl. Phys. 51, 185301 (2018).

[36] S. Mukherjee, P. Priyadarshi, A. Sharma, and B. Muralidharan, IEEE Trans. Electron Devices 65, 1896 (2018).

[37] S. Mukherjee and B. Muralidharan, Phys. Rev. Appl. 12, 024038 (2019).

[38] S. Singh, K. Thakar, N. Kaushik, B. Muralidharan, and S. Lodha, Phys. Rev. Appl. 10, 014022 (2018).

[39] A. Singha and B. Muralidharan, Sci. Rep. 7, 7879 (2017).

[40] A. Singha and B. Muralidharan, J. Appl. Phys. 124, 144901 (2018).

[41] A. Sinha, A. Sharma, A. Tulapurkar, V. R. Rao, and B. Muralidharan, Phys. Rev. Mater. 3, 124005 (2019).

[42] M. Huang, T. A. Pascal, H. Kim, W. A. Goddard, and J. R. Greer, Nano Lett. 11, 1241 (2011).

[43] A. D. Smith, F. Niklaus, A. Paussa, S. Schröder, A. C. Fischer, M. Sterner, S. Wagner, S. Vaziri, F. Forsberg, D. Esseni, M. Östling, and M. C. Lemme, ACS Nano 10, 9879 (2016). 
[44] A. Mawrie and B. Muralidharan, Phys. Rev. B 99, 075415 (2019).

[45] A. Mawrie and B. Muralidharan, Phys. Rev. B 100, 081403(R) (2019).

[46] N. M. R. Peres, J. M. B. Lopes dos Santos, and T. Stauber, Phys. Rev. B 76, 073412 (2007).

[47] R. M. Ribeiro, V. M. Pereira, N. M. R. Peres, P. R. Briddon, and A. H. C. Neto, New J. Phys. 11, 115002 (2009).

[48] Y. Hasegawa, R. Konno, H. Nakano, and M. Kohmoto, Phys. Rev. B 74, 033413 (2006).

[49] V. M. Pereira, A. H. Castro Neto, and N. M. R. Peres, Phys. Rev. B 80, 045401 (2009).

[50] M. Farjam and H. Rafii-Tabar, Phys. Rev. B 80, 167401 (2009).

[51] S.-M. Choi, S.-H. Jhi, and Y.-W. Son, Phys. Rev. B 81, 081407(R) (2010).

[52] Z. H. Ni, T. Yu, Y. H. Lu, Y. Y. Wang, Y. P. Feng, and Z. X. Shen, ACS Nano 3, 483 (2009).

[53] M. Huang, H. Yan, T. F. Heinz, and J. Hone, Nano Lett. 10, 4074 (2010).

[54] C. Jeong, R. Kim, M. Luisier, S. Datta, and M. Lundstrom, J. Appl. Phys. 107, 023707 (2010).
[55] N. W. Ashcroft, N. D. Mermin, and D. Wei, Solid State Physics (Cengage Learning, Boston, 2016).

[56] K. Nomura and A. H. MacDonald, Phys. Rev. Lett. 98, 076602 (2007).

[57] L.-D. Landau and E.-M. Lifshitz, Course Theor. Phys. 7, 32 (1986).

[58] A. Boisen, S. Dohn, S. S. Keller, S. Schmid, and M. Tenje, Rep. Prog. Phys. 74, 036101 (2011).

[59] V. Seena, A. Fernandes, P. Pant, S. Mukherji, and V. R. Rao, Nanotechnology 22, 295501 (2011).

[60] I. C. Nelson, D. Banerjee, W. J. Rogers, and M. S. Mannan, in Micro (MEMS) and Nanotechnologies for Space Applications (International Society for Optics and Photonics, Bellingham, WA, 2006), Vol. 6223, p. 622300.

[61] L. Pinnaduwage, A. Wig, D. Hedden, A. Gehl, D. Yi, T. Thundat, and R. Lareau, J. Appl. Phys. 95, 5871 (2004).

[62] J. Wang, S. Deng, Z. Liu, and Z. Liu, Natl. Sci. Rev. 2, 22 (2015).

[63] D. Malko, C. Neiss, F. Vines, and A. Görling, Phys. Rev. Lett. 108, 086804 (2012).

[64] B. K. Sharma and J.-H. Ahn, Solid-State Electron. 89, 177 (2013). 